\title{
Aspectos relacionados à saúde do jovem escolar: uma revisão
}

\author{
Aspects related to health of young schools: a review
}

Aspectos relacionados con la salud de las escuelas jóvenes: una revisión

Diogo Queiroz Allen Palacio ${ }^{1,2 *}$, Antonio Germane Alves Pinto ${ }^{3}$, Paulo Gabriel Lima da Rocha ${ }^{2}$, Stela Lopes Soares ${ }^{4,5}$, Iriadelia Soraya Ribeiro Rabelo², Ágata Cristina Marques Aranha².

\section{RESUMO}

Objetivo: Rever sistematicamente a literatura, procurando evidências científicas sobre a condição de saúde dos estudantes pelo mundo. Utilizou-se como modelo as diretrizes dos Principais Itens Para Relatar Revisões Sistemáticas e Meta-análises (PRISMA). Métodos: Buscou-se artigos publicados em inglês, espanhol e português, com um corte temporal de cinco anos (2015 a 2019), em cinco base de dados: Web of Science, Science Direct, Scopus, Pubmed e Lilacs. Resultados: No final, restaram para a análise dos resultados 39 artigos, esses foram divididos em categorias considerando suas afinidades temáticas. Em todas as categorias, os estudos trazem indicadores de prevalência de aspectos relacionados com a saúde que alertam para os perigos e agravos ao bem-estar do estudante adolescente. Considerações finais: Deduz-se que as atuais medidas de promoção de saúde no âmbito escolar não estão sendo satisfatórias. As pesquisas elencadas nesta revisão já apontam para a urgência no desenvolvimento de programas educacionais que melhorem a qualidade de vida dos estudantes e assim se evite possíveis complicações ao setor de saúde pública.

Palavras-chave: Saúde, Adolescente, Instituições acadêmicas.

\begin{abstract}
Objective: To systematically review the literature, looking for scientific evidence about the health condition of students around the world. The guidelines of the Main Items for Reporting Systematic Reviews and Metaanalyzes (PRISMA) were used as a model. Methods: We searched for articles published in English, Spanish and Portuguese, with a five-year time span (2015 to 2019), in five databases: Web of Science, Science Direct, Scopus, Pubmed and Lilacs. Results: In the end, 39 articles remained for the analysis of the results, these were divided into categories considering their thematic affinities. In all categories, the studies bring indicators of prevalence of aspects related to health that alert to the dangers and aggravations to the well-being of the adolescent student. Final considerations: It appears that the current health promotion measures at school are not being satisfactory. The researches listed in this review already point to the urgency in the development of educational programs that improve the quality of life of students and thus avoid possible complications to the public health sector.
\end{abstract}

Keywords: Health, Adolescent, Schools.

${ }^{1}$ Secretária de Educação de Fortaleza (SEDUC), Fortaleza - CE. *E-mail: diogoallenpalacio@gmail.com

2 Universidade de Trás-os-Montes e Alto Douro (UTAD), Vila Real, Portugal.

${ }^{3}$ Universidade Regional do Cariri (URCA), Crato - CE.

${ }^{4}$ Universidade Estadual do Ceará (UECE), Fortaleza - CE.

${ }^{5}$ Centro Universitário INTA (UNINTA), Sobral - CE.

SUBMETIDO EM: 3/2021

ACEITO EM: 3/2021

PUBLICADO EM: 4/2021 


\section{RESUMEN}

Objetivo: Revisar sistemáticamente la literatura, buscando evidencia científica sobre el estado de salud de los estudiantes alrededor del mundo. Se utilizaron como modelo las directrices de los Elementos principales para la presentación de informes de revisiones sistemáticas y metanálisis (PRISMA). Métodos: Se buscaron artículos publicados en inglés, español y portugués, con un lapso de tiempo de cinco años (2015 a 2019), en cinco bases de datos: Web of Science, Science Direct, Scopus, Pubmed y Lilacs. Resultados: Al final quedaron 39 artículos para el análisis de los resultados, estos fueron divididos en categorías considerando sus afinidades temáticas. En todas las categorías, los estudios traen indicadores de prevalencia de aspectos relacionados con la salud que alertan sobre los peligros y agravamientos para el bienestar del estudiante adolescente. Consideraciones finales: Parece que las medidas actuales de promoción de la salud en la escuela no están siendo satisfactorias. Las investigaciones enumeradas en esta revisión ya apuntan a la urgencia en el desarrollo de programas educativos que mejoren la calidad de vida de los estudiantes y así evitar posibles complicaciones al sector de la salud pública.

Palabras clave: Salud, Adolescente, Instituciones académicas.

\section{INTRODUÇÃO}

A fase da adolescência caracteriza-se como um período vital entre a infância e a fase adulta. É nesse período que o indivíduo vivencia suas principais mudanças corporais, a aquisição pôndero-estatural e fatores sexuais secundários se consolidam. No sexo feminino, temos o advento da menarca selando o início do ciclo reprodutivo e consequentemente complexas transformações que repercutem no desenvolvimento cognitivo, ampliando-se para descoberta de novas relações interpessoais, intrapessoais e com o meio (FAIAL LCM, et al., 2016).

É também no período da adolescência que se desperta o espírito da autonomia, onde o jovem tende a se tornar mais sujeito a comportamentos de risco à sua saúde. O uso abusivo de álcool e drogas ilícitas, o tabagismo, violência, sedentarismo e hábitos alimentares inadequados, são exemplos de condutas comuns aos adolescentes. Salienta-se ainda a expressividade e significância que a juventude possui como geração futura, tornando pertinente discussões com o foco na redução dos agravos à sua saúde, considerando o potencial existente para problemas no setor de saúde pública (Maia CMMSC, et al., 2017).

Referenciando a Organização Mundial de Saúde, Sales JKD, et al. (2020) afirma que a fase da adolescência corresponde ao período transcorrido dos 10 aos 19 anos de idade, em que os mesmos se encontram na escola.

É na escola que o adolescente em formação passa parte considerável do seu dia, onde desenvolve sua socialização com o próximo, estreitando vínculos, troca de experiências e difusão de conhecimento. $O$ ato de ensinar e aprender desenvolve o senso crítico no aluno o despertando para sua responsabilidade social e participação ativa nas práticas de promoção de saúde. Com essa perspectiva, a exploração do espaço educacional tem sido apontada como o caminho para se aprimorar as ações de saúde efetivas e adequadas aos anseios do público jovem (CLAUDIA CPSR, et al., 2016).

Antes de se pensar nos meios ou possibilidades de ações, é vital o processo de diagnóstico da atual situação ou condição de saúde de um público-alvo. No espaço escolar especificamente, é o constante monitoramento sobre o estado de saúde do estudante que vai nortear quais as futuras medidas, além de observar os resultados de ações já executadas (DE SOUZA DS, et al., 2018).

O ministério da saúde declara que os adolescentes e jovens constituem um grupo populacional que exige novos modos de produzir saúde, no qual o ciclo de vida particularmente saudável, evidencia que os agravos em saúde decorrem, em grande medida, de modos de fazer "andar a vida", de hábitos e comportamentos, 
que, em determinadas conjunturas, os vulnerabilizam. As vulnerabilidades produzidas pelo contexto social e as desigualdades resultantes dos processos históricos de exclusão e discriminação determinam os direitos e as oportunidades de adolescentes e jovens brasileiros (BRASIL, 2018).

A adolescência, então, pode representar um período crítico, onde vários hábitos e comportamentos são estabelecidos. Os comportamentos de risco adotados pelos adolescentes podem ser compreendidos como atividades que possam colocar em risco à saúde. Essa forma de entender, agir e se posicionar frente à realidade é influenciada pelo contexto familiar e social, como escola, comunidade, amigos e a mídia (BARBOSA FMN, et al., 2016).

É importante compreender, que a realidade em que vivemos está em constante alteração, seja de natureza social, política, econômica ou geográfica, o que implica em mudanças comportamentais ou no estilo de vida da população. Deve-se considerar ainda, a heterogeneidade inerente à pluralidade cultural específica a cada região, o que nos permite afirmar que as carências de saúde alternam de acordo com a realidade local. Todas essas constantes alterações repercutem como um efeito cascata para dentro do espaço escolar, se considerarmos que cada escola costuma ser reflexo do contexto que a cerca (MAIA CMMSC, et al., 2017).

Assim, as demandas de saúde coletivas e individuais dos estudantes, devem ter suas peculiaridades respeitadas para que planos de ações efetivos sejam formulados. Mais uma vez, tratamos o monitoramento dos aspectos relacionados à saúde do jovem escolar como um pré-requisito para se pensar na eficiência e eficácia sobre as práticas de promoção de saúde na escola (FAIAL LCM, et al., 2016).

A presente revisão sistemática justifica-se pela necessidade de se conhecer com profundidade como as produções científicas têm monitorado as informações relacionadas ao estado de saúde do estudante. Haja vista, a possibilidade de se delimitar planos ou medidas de ações compatíveis com as necessidades sociais desta comunidade. Assim, o objetivo do presente estudo foi rever sistematicamente a literatura, procurando evidências científicas sobre a condição de saúde dos estudantes pelo mundo, considerando o contexto social moderno do estilo de vida do jovem escolar.

\section{MÉTODOS}

Para a construção do presente estudo, utilizou-se como modelo as diretrizes dos Principais Itens Para Relatar Revisões Sistemáticas e Meta-análises (PRISMA), sendo a sumarização das antigas diretrizes de ensaios clínicos randomizados e relatos de Meta-análise da recomendação da Qualidade dos Relatos de Meta-análises (QUORUM). Os conceitos desse novo modelo são relevantes para a formulação de uma revisão sistemática clara e objetiva, com métodos sistemáticos que favorece a seleção de pesquisas importantes, sob um olhar crítico e reflexivo (GALVÃO TF, et al., 2015).

Para atender os critérios de declaração de Prisma, dois avaliadores buscaram, de forma independente, artigos publicados em inglês, espanhol e português, com um corte temporal de cinco anos (2015 a 2019), em cinco base de dados: Web of Science, Science Direct, Scopus, Pubmed e Literatura Latino-Americana e do Caribe em Ciências da Saúde (Lilacs). Os termos de busca foram aplicados em todas as bases de dados, sendo eles: "School", "School Health", "Health", "Public School", "Student", "Adolescent" e "Health of Adolescent" com as seguintescombinações: "School" AND "Public" AND "Health" AND "Student", "School Health" AND "Public School", "School Health" AND "Student", "Public School" AND "Health" AND "Adolescent" e "Health of Adolescent" AND "School". Aplicou-se como filtro de buscas, a presença dos termos somente nos títulos e resumos dos trabalhos acadêmicos, o que favoreceu a seleção de artigos com afinidade temática a presente pesquisa.

O processo de seleção dos artigos se deu a partir de uma sequência de passos: Identificação, Triagem, Elegibilidade e Inclusão. Os critérios adotados para inclusão e exclusão dos artigos foram: (a) O cenário da pesquisa precisa ser as Instituições de Ensino; (b) Participantes são estudantes do ensino médio e acima de 14 anos; (c) Deve-se necessariamente incluir escolas públicas. As buscas foram realizadas entre março e abril de 2020. A Figura 1 a seguir detalha o quantitativo dos artigos durante o processo de seleção: 
Figura 1 - Processo de seleção dos artigos nas bases de dados.
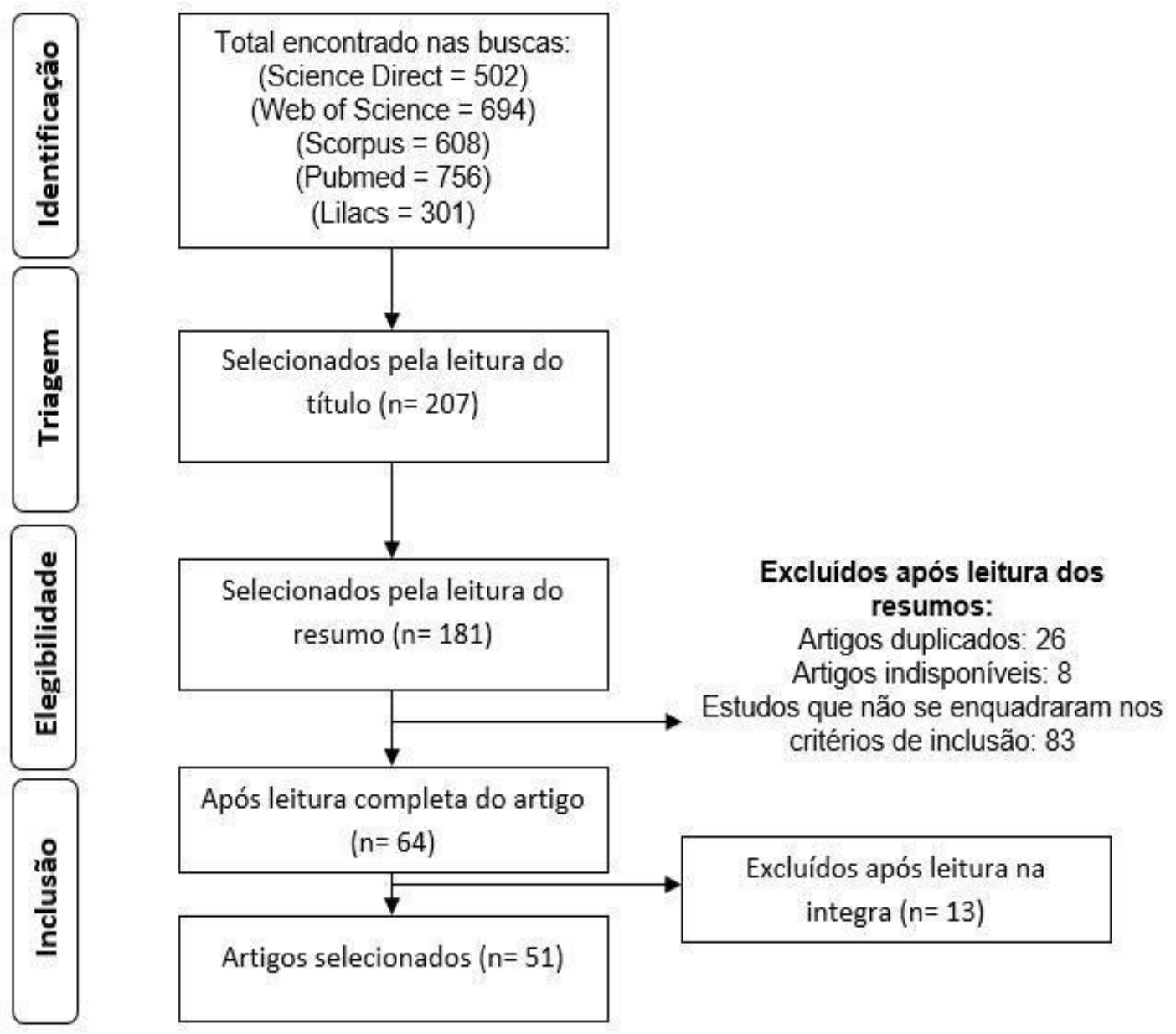

Fonte: Palacio DQA, et al., 2021.

A qualidade metodológica dos estudos revisados foi avaliada de forma independente por dois revisores, utilizando uma lista de verificação com vários critérios em alinhamento com as diretrizes da iniciativa denominada Strengthening the Reporting of Observational in Epidemiology (STROBE). Especificamente utiliza-se uma lista de verificação que contém 22 itens, com recomendações sobre o que deveria ser incluído em uma descrição mais precisa e completa de estudos observacionais (MALTA M, et al., 2010).

Durante o processo de avaliação de forma independente dos revisores sobre os artigos, estes foram classificados como "critérios atendidos" e "critérios não atendidos". Em casos de divergência entre os revisores, um terceiro revisor foi consultado para se atingir um consenso. Dessa forma, 12 artigos foram excluídos por não atender os critérios de STROBE. No final, restaram para a análise dos resultados 39 artigos, apresentados na sessão a seguir.

\section{RESULTADOS E DISCUSSÂO}

Dentro dos métodos de revisão sistemáticas, ao se investigar fontes cientificas de forma quantitativa, pesquisadores precisam saber como se dá a produção, publicação, consulta e replicação das informações em determinadas áreas de conhecimentos. Especificamente nos trabalhos científicos dentro da grande área da saúde, é relevante se descrever mais do que o "quanto" se produz, e estender a descrição ao alcance de se compreender "como" as produções vem se desenvolvendo (CAMARGO FC, et al., 2017).

Os estudos selecionados, tiveram diversos cenários de aplicação, retratando vários contextos diferentes sobre a saúde do estudante adolescente, sendo detalhado no Gráfico 1 a seguir: 
Gráfico 1 - Descrição do quantitativo de estudos por continente.

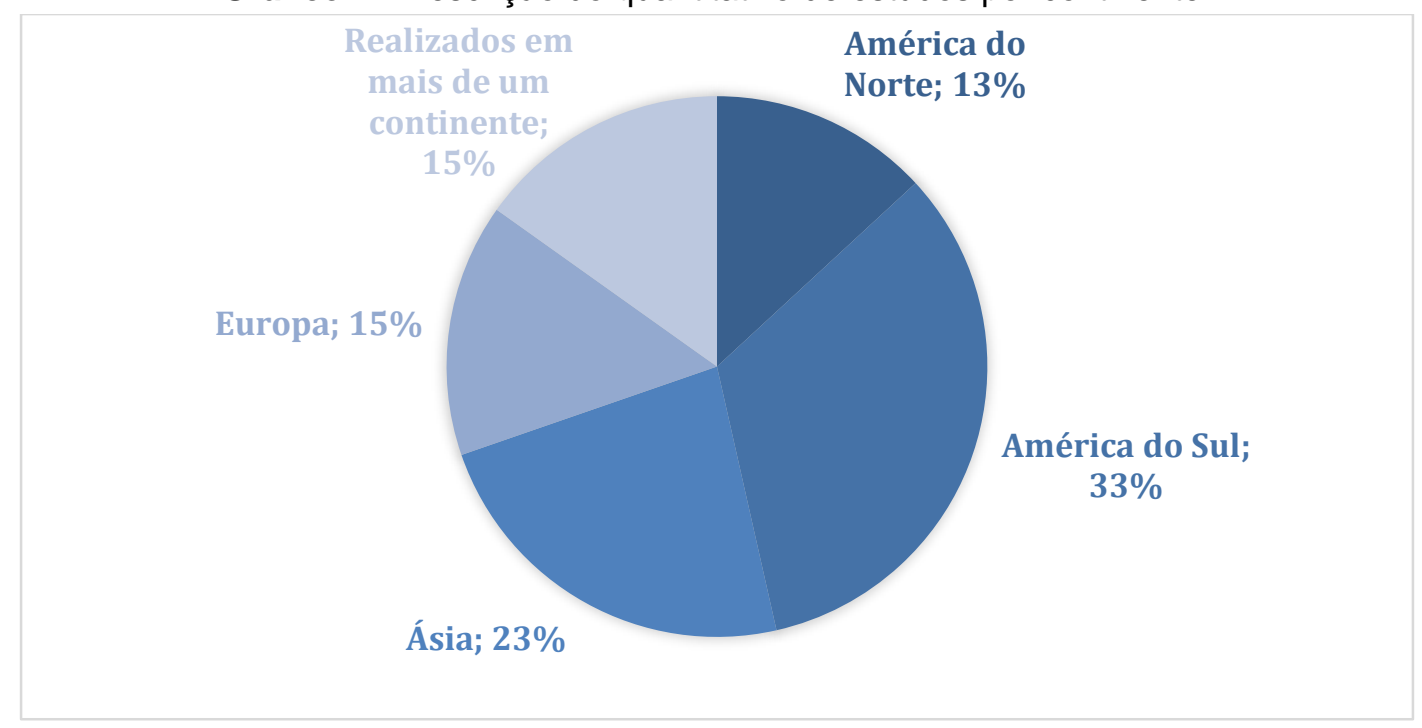

Fonte: Palacio DQA, et al., 2021.

Organizando ou agrupando os locais dos estudos por continentes temos o seguinte quantitativo: América do Norte: cinco (13\%), América do Sul: 13 (33\%), Europa: seis (15\%) e Ásia: nove (23\%). Nos continentes Oceania, África e Antártica não houve trabalhos selecionados. E ainda, seis estudos (15\%) foram realizados em vários países se enquadrando em mais de um continente.

No que se refere ao desenho metodológico dos estudos, todos os trabalhos foram caracterizados como "estudos epidemiológicos de corte transversal", com abordagem quantitativa na coleta, análise e apresentação dos dados. O Gráfico 2 a seguir apresenta os instrumentos utilizados pelos estudos na coleta de dados:

Gráfico 2 - Instrumentos utilizados pelos estudos no processo de coleta de dados.

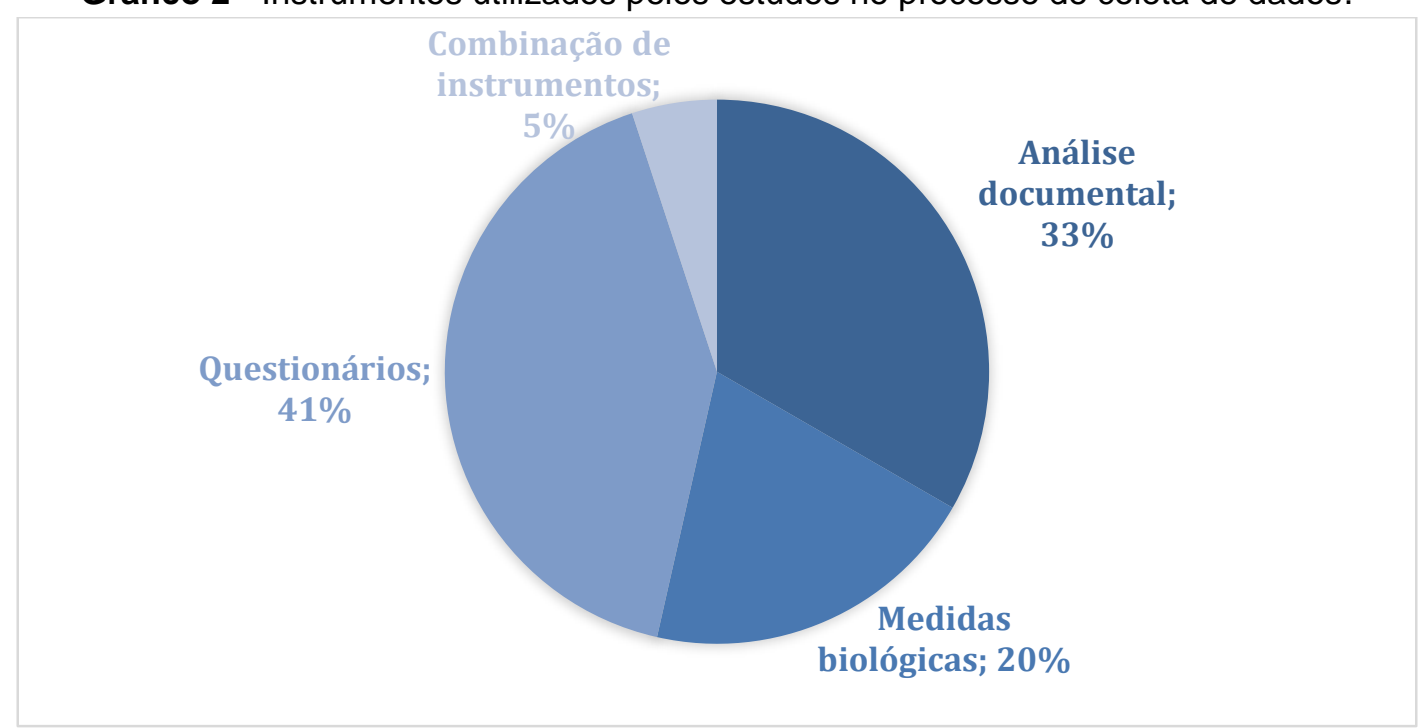

Fonte: Palacio DQA, et al., 2021.

O processo de coleta de dados nos artigos, ocorreu de quatro formas: (a) pesquisas de análise documental, a partir de acessos a bases de dados internacionais e nacionais, representando $33 \%$ do total, (b) medidas biológicas como testes físicos e variáveis antropométricas, correspondendo $20 \%$ dos estudos, (c) Questionários, sendo esses validados, adaptados e pilotos, confere o instrumento de maior utilização pelos estudos $41 \%$ e (d) artigos que utilizaram a combinação entre os instrumentos já citados, equivalendo a $5 \%$. 
Diante da quantidade final de artigos selecionados (39), optou-se aqui por categorizar os trabalhos a partir de suas afinidades temáticas. Um total de cinco categorias foram criadas, com base nos seguintes critérios apresentadas no Quadro 1 a seguir:

Quadro 1 - Categorias e seus respectivos critérios de inclusão.

\begin{tabular}{|c|l|}
\hline Categorias / Agrupamento temático & Critérios e descrição dos agrupamentos temáticos \\
\hline $\begin{array}{c}\text { Saúde mental, aspectos psicossociais e } \\
\text { neurológicos (SM) }\end{array}$ & $\begin{array}{l}\text { Artigos que se destinassem a apresentar informações } \\
\text { referentes ou associadas ao estado de saúde mental do } \\
\text { jovem escolar, incorporando aspectos psicossociais e } \\
\text { neurofisiológicos. }\end{array}$ \\
\hline $\begin{array}{c}\text { Aspectos nutricionais e práticas corporais } \\
\text { (ANPC) }\end{array}$ & $\begin{array}{l}\text { Artigos que apresentem dados de prevalência sobre } \\
\text { aspectos nutricionais e práticas corporais (atividade } \\
\text { física, exercício físico e práticas esportivas). }\end{array}$ \\
\hline $\begin{array}{c}\text { Indicadores biológicos de saúde ou } \\
\text { biomarcadores (BIO) }\end{array}$ & $\begin{array}{l}\text { Artigos dentro do seguimento biologicista, que } \\
\text { apresentam dados sobre o estado de saúde do jovem } \\
\text { escolar, a partir da quantificação de marcadores } \\
\text { biológicos de saúde ou biomarcadores. }\end{array}$ \\
\hline $\begin{array}{c}\text { Aspectos de natureza comportamental } \\
\text { associados a saúde (COMP) }\end{array}$ & $\begin{array}{l}\text { Artigos que se destinam a apresentar dados de } \\
\text { prevalência sobre os aspectos relacionados aos hábitos } \\
\text { e comportamentos do estudante que representam fatores } \\
\text { de riscos para sua saúde. }\end{array}$ \\
\hline Prevalência de vacinação, uso de \\
medicamentos e consumo de drogas (MED)
\end{tabular}

Fonte: Palacio DQA, et al., 2021.

O Quadro 2, sintetiza os principais resultados encontrados nos artigos, assim como as indicações e proposições que são apontadas pelos autores para que se tenha um impacto social significativo. Ao se analisar os estudos relacionados à saúde mental do jovem escolar percebe-se o destaque de alguns dados. No estudo de Baldursdottir B, et al. (2017) com adolescentes do ensino médio, foi identificado que $26 \%$ relataram exposição ao bullying nos últimos 30 dias, e que um a cada três estudantes foram vítimas de violência física no último ano.

Vancampfort $D$, et al. (2018), relata que a prevalência de sintomas depressivos entre os estudantes é de $28,7 \%$, e Badr HE, et al. (2018) constata algo semelhante descrevendo que entre os participantes de seu estudo 49,6\%,63\% e 43,8\% relataram ter depressão, ansiedade e estresse, respectivamente. Em decorrência de todos esses indicadores elevados, Benbernishty $R$, et al. (2018) indica que as taxas de ideação suicida entre os jovens variam entre $4 \%$ e $67 \%$, com mediana de $19,3 \%$ e média de $20 \%$.

Podemos compreender que durante a adolescência, alguns transtornos da saúde mental podem ocorrer e devem ser detectados precocemente para uma intervenção eficaz. Alguns problemas envolvem também doenças sistêmicas e, portanto, faz-se necessária uma abordagem multidisciplinar para um manejo adequado (BRASIL, 2018).

De acordo com O'Reilly M, et al. (2018) existe um desenvolvimento e esforço para a implementação de intervenções na saúde mental de escolares. Porém, a eficácia dessas intervenções, precisa ser melhor avaliada. Para os autores, é necessária uma compreensão maior sobre os métodos de promoção da saúde na escola para alcançar sucesso nessas intervenções. Isso posto, é evidente a gravidade da condição de bem-estar social do jovem escolar, esses indicadores devem motivar práticas de intervenção no âmbito da escola, principalmente com práticas de educação em saúde, que disparem a conscientização do estudante sobre os diversos fatores que impactam em sua saúde mental.

Vancampfort $D$, et al. (2019), destaca ainda que na atualidade, carecemos de pesquisas longitudinais que favorecem a compreensão dos processos de adoecimento, onde sobre uma outra ótica, pode potencializar as intervenções. 
Quadro 2 - Síntese dos resultados dos artigos selecionados na revisão sistemática.

\begin{tabular}{|c|c|c|c|}
\hline Categorias temáticas & Autores & Principais resultados & Indicações e proposições \\
\hline $\begin{array}{l}\text { SM - Saúde mental, aspectos } \\
\text { psicossociais e neurológicos }\end{array}$ & $\begin{array}{l}\text { Guo L, et al. (2016), Vancampfort D, et } \\
\text { al. (2018), Yang L, et al. (2017), } \\
\text { Baldursdottir B, et al. (2017), Beckman } \\
\text { L, et al. (2016), Badr HE, et al. (2018), } \\
\text { Benbenishty R, et al. (2018), } \\
\text { Vancampfort D, et al. (2019). } \\
\text { Albuhairan F, et al. (2017). }\end{array}$ & $\begin{array}{l}\text { A depressão, o bullying e a violência, são na atualidade sérios } \\
\text { problemas de saúde pública. As pesquisas evidenciam que } \\
\text { com o aumento da idade, os sintomas depressivos } \\
\text { aumentam, atingindo predominantemente os estudantes do } \\
\text { ensino médio. A ansiedade e o stress estão cada vez mais } \\
\text { presentes entre os jovens, assim como, as taxas de ideação } \\
\text { suicida. Os adolescentes do sexo masculino apresentam } \\
\text { maiores fatores de risco para depressão em detrimento dos } \\
\text { maus hábitos comportamentais. }\end{array}$ & $\begin{array}{l}\text { Os estudos alertam para a necessidade de programas } \\
\text { educacionais para que se alcance uma conscientização } \\
\text { dos jovens escolares sobre os fatores que circundam a } \\
\text { saúde mental destes. E ainda, torna-se preciso pensar } \\
\text { nos alunos que já se encontram em condição crítica no } \\
\text { que se refere ao bem-estar emocional, com suporte de } \\
\text { tratamento aos possíveis traumas instalados. }\end{array}$ \\
\hline $\begin{array}{c}\text { ANPC - Aspectos nutricionais } \\
\text { e práticas corporais }\end{array}$ & $\begin{array}{l}\text { Assis MM, et al. (2017), Chaves OC, } \\
\text { et al. (2018), Minatto G, et al. (2016), } \\
\text { Alsubaie AS e Omer EO (2015), } \\
\text { Sharma B, et al. (2018), Santos SJD, } \\
\text { et al. (2015), Khan A, et al. (2017), } \\
\text { Silva FMD, et al. (2016). }\end{array}$ & $\begin{array}{l}\text { A maioria dos adolescentes nos estudos foram identificados } \\
\text { como insuficientemente ativos. As meninas costumam } \\
\text { apresentar aptidão física geral inferior à dos meninos. Os } \\
\text { estudantes tendem a fazer menos atividade física com o } \\
\text { avanço da idade. A prevalência do consumo inadequado de } \\
\text { frutas e hortaliças entre os adolescentes foi alta. Uma parcela } \\
\text { significativa dos estudantes relata ingerir refrigerantes } \\
\text { diariamente e baixa ingestão de vitamina D. }\end{array}$ & $\begin{array}{l}\text { Os estudos consideram os dados apresentados como } \\
\text { preocupantes pelo grande risco a saúde dos jovens. } \\
\text { Alertam para o incentivo à prática de atividade física no } \\
\text { tempo destinado ao lazer e a conscientização dos pais. } \\
\text { O espaço escolar é frisado como um cenário } \\
\text { fundamental para melhoria dos indicadores, } \\
\text { principalmente através das aulas de Educação Física. } \\
\text { No que tange a alimentação, sugere-se a redução do } \\
\text { consumo de açúcar e aumento da ingestão de vitaminas } \\
\text { e saís minerais por controle da alimentação dos } \\
\text { estudantes na escola. }\end{array}$ \\
\hline $\begin{array}{l}\text { BIO - Indicadores biológicos } \\
\text { de saúde ou biomarcadores }\end{array}$ & $\begin{array}{l}\text { Yang L, et al. (2019), Garcia- } \\
\text { Continente X, et al. (2015), Musa TH, } \\
\text { et al. (2016), Jardim, et al. (2018), } \\
\text { Frank R, et al. (2018), Nascimento BR, } \\
\text { et al. (2016), Kaestner TL, et al. } \\
\text { (2018). }\end{array}$ & $\begin{array}{l}\text { Os dados dos estudos confirmam a carga dupla contínua de } \\
\text { baixo peso e excesso de peso entre adolescentes de países } \\
\text { de baixa e média renda. A magnitude do excesso de peso e } \\
\text { obesidade reflete um problema de saúde pública. A Doença } \\
\text { reumática cardíaca continua sendo uma condição importante } \\
\text { e pouco reconhecida entre os adolescentes. Identificou-se } \\
\text { prevalência significativa de colesterol sugestivos entre os } \\
\text { estudantes. }\end{array}$ & $\begin{array}{l}\text { As pesquisas apontam a necessidade de planos e } \\
\text { políticas que abordem as condições relatadas. } \\
\text { Programas específicos de combate ao sobrepeso e } \\
\text { obesidade são importantes para a saúde na escola. Os } \\
\text { dados de monitoramento contribuem para o argumento } \\
\text { convincente de investimentos renovados em práticas } \\
\text { preventivas entre os jovens estudantes. }\end{array}$ \\
\hline $\begin{array}{c}\text { COMP - Aspectos de natureza } \\
\text { comportamental associados a } \\
\text { saúde }\end{array}$ & $\begin{array}{l}\text { Vilela TDS, et al. (2016), Al Subait AA, } \\
\text { et al. (2016), Jamir L, et al. (2019), } \\
\text { Fonseca CD, et al. (2015), Amaral } \\
\text { MOP, et al. (2017), Meyer MKH, et al. } \\
\text { (2017), Lu L, et al. (2018), Sampasa- } \\
\text { Kanyinga H, et al. (2018), Al-Qurashi } \\
\text { H, et al. (2016). }\end{array}$ & $\begin{array}{l}\text { O déficit de sono é tratado pelos estudos como uma realidade } \\
\text { hoje entre os jovens. Uma parcela considerável dos } \\
\text { estudantes não está satisfeita com a aparência dos seus } \\
\text { dentes, e apresentam sintomas de má higienização bucal. O } \\
\text { uso abusivo/ dependência da internet é um fator } \\
\text { corriqueiramente identificado pelos estudos. }\end{array}$ & $\begin{array}{l}\text { As pesquisas sugerem que programas educacionais } \\
\text { devem ser urgentemente desenvolvidos para melhorar a } \\
\text { qualidade de vida dos estudantes e evitar possíveis } \\
\text { complicações mais graves ao setor de saúde pública. }\end{array}$ \\
\hline $\begin{array}{c}\text { MED - Prevalência de } \\
\text { vacinação, uso de } \\
\text { medicamentos e consumo de } \\
\text { drogas }\end{array}$ & $\begin{array}{l}\text { Szilagyi PG, et al. (2018), Baiden P, et } \\
\text { al. (2019), Jampel JD, et al. (2016), } \\
\text { Millarini V, et al. (2019), Anand V, et al. } \\
\text { (2015), Zander NC, et al. (2018). }\end{array}$ & $\begin{array}{l}\text { A taxa de vacinação contra o Inlfuenza vírus tem aumentado } \\
\text { nas escolas. Os adolescentes têm iniciado o consumo de } \\
\text { álcool de forma cada vez mais prematura. O consumo de } \\
\text { anabolizantes tem sido comum entre meninos que almejam } \\
\text { ganhar massa muscular e meninas que desejam emagrecer. } \\
\text { A experimentação do tabaco pelos adolescentes está sendo } \\
\text { considerada um grande fator de risco para saúde. Mais da } \\
\text { metade dos alunos que compreendem amostra dos estudos } \\
\text { já utilizaram cigarro eletrônico. }\end{array}$ & $\begin{array}{l}\text { Os estudos relatam que as práticas educativas no } \\
\text { espaço escolar relacionadas ao consumo de drogas não } \\
\text { estão sendo eficazes, necessitando assim se repensar } \\
\text { as medidas de ação sobre os jovens. } \\
\text { O combate ao tabagismo já é considerado um aspecto } \\
\text { associado ao setor de saúde pública, dessa forma, as } \\
\text { práticas preventivas devem ser potencializadas. }\end{array}$ \\
\hline
\end{tabular}

Fonte: Palacio DQA, et al., 2021. 
No que tange os artigos relacionados aos aspectos nutricionais e práticas corporais, elencamos os seguintes resultados chaves: Alsubaie AS e Omer EO (2015) descrevem em seu estudo com estudantes, que embora $36,4 \%$ alegam que praticam exercícios físicos suficientes durante a semana, apenas, $15,5 \%$ de fato estavam praticando atividade física em um nível recomendado. Em estudo mais recente, Sharma $\mathrm{B}$, et al. (2018), os dados encontrados são semelhantes, foi diagnosticado que $78 \%$ dos adolescentes não cumpriram a recomendação global da Organização Mundial da Saúde sobre atividade física na última semana antes da pesquisa. Santos SJD, et al. (2015), complementa com os resultados de seu estudo com $65,1 \%$ dos adolescentes sendo classificados como insuficientemente ativos e $64,9 \%$ referiram não frequentar as aulas de Educação Física.

A alimentação saudável ultrapassa a ingestão de nutrientes, referindo-se também aos alimentos específicos que fornecem os nutrientes, bem como às inúmeras combinações possíveis a partir deles e as dimensões sociais e culturais do ato de comer. Além de prevenir ou causar doenças, a alimentação afeta a identidade, o sentimento de pertencimento social, o estado de humor, o prazer, a aptidão, a autonomia e várias outras dimensões centrais do estado de bem-estar das pessoas (BRASIL, 2014).

Silva FMDA, et al. (2018), trazem em sua pesquisa a prevalência de $88,6 \%$ de consumo inadequado de frutas e hortaliças entre os estudantes. Chaves OC, et al. (2018), complementa trazendo o indicador de que $21,7 \%$ dos estudantes relatam o consumo de refrigerantes diariamente.

A partir dos principais resultados dos estudos recém expostos, fica evidente o quanto é preocupante 0 contexto pelo grande risco à saúde dos jovens. O elevado consumo de açúcar, somado a baixa ingestão de nutrientes e adesão a práticas de atividades físicas devem ser elementos de constante discussão no âmbito escolar.

Khan A, et al. (2017), explicam que algumas medidas simples como: fornecer material esportivo, estimular a caminhada para a escola, apoio e exemplo paternal sobre as práticas, são medidas tangíveis ao aumento do envolvimento dos estudantes com hábitos de vida mais saudáveis.

Para Souza AA e Cadete MMM (2017) é importante refletir sobre o valor da família e da escola na escolha de hábitos saudáveis entre os estudantes. Em relação à alimentação, enfatizam que estes hábitos são influenciados por questões emocionais, culturais, familiares e sociais, com grandes repercussões nas mudanças comportamentais. Nesse sentido, "torna-se imperiosa a adoção de estratégias capazes de municiar familiares, crianças e a comunidade escolar para que, em conjunto, trabalhem educativamente e mudem o panorama atual"

Ao se discutir sobre os indicadores biológicos de saúde, trazemos inicialmente o estudo de GarciaContinente $X$, et al. (2015), onde foi encontrado uma prevalência de excesso de peso de $26,1 \%$ nos meninos ( $6,2 \%$ de obesidade) e $20,6 \%$ nas meninas $(3,7 \%$ de obesidade), observando ainda neste estudo que 0 excesso de peso esteve associado ao aumento da idade. Jardim TV, et al. (2018), afirma que na atualidade a maioria dos estudantes sem excesso de peso, são encontrados nas escolas públicas $84,2 \%$. Ainda sobre biomarcadores, Kaestner TL, et al. (2018), estima em seu estudo que aproximadamente 100.000 (1 em cada 200) adolescentes brasileiros com idade entre 12 e17 anos sejam suspeitos de ter hipercolesterolemia familiar com base nos níveis de níveis LDLc (Low Density Lipoproteins) e não-HDLc (Low Density Lipoproteins).

Frank R, et al. (2018), afirma que atualmente os adolescentes de ambos os sexos, que percebem seu peso corporal abaixo do esperado, realizam mais iniciativas para ganhar peso, e aqueles que percebem seu peso como acima do ideal fazem mais esforços para perder. Isso pode fundamentar ações no espaço escolar, medidas dentro da saúde coletiva dos jovens, apresentando seus próprios biomarcadores de saúde para interpretação e compreensão do processo de adoecimento, assim os estudantes se tornam protagonistas no cuidado e manutenção do seu bem-estar fisiológico.

É possível afirmar que o aumento significativo nas prevalências de obesidade em adolescentes tem se tornado irrefutável, apontando para a necessidade de medidas preventivas, uma vez que este grupo está 
diretamente exposto a ambientes e hábitos favoráveis a esta condição. Outro componente fundamental para os debates sobre saúde do jovem escolar, são seus hábitos e comportamentos, nesse sentido, iniciamos a descrição dos resultados chave dos estudos analisados (DO NASCIMENTO FJ, 2020).

Na pesquisa de Vilela TDS, et al. (2016), foi constatado que pelo menos $39 \%$ dos adolescentes estavam com privação de sono. Jamir $L$, et al. (2019), complementa, afirmando que $30,3 \%$ dos participantes de sua pesquisa preenchiam os critérios de dependência sobre equipamentos eletrônicos (computadores, celulares e videogames).

Esses dois marcadores de prevalência são muito relevantes, por retratar uma discussão atual, ou seja, descreve-se aqui os reflexos da interação do estudante com o mundo moderno. Para Amaral MOP, et al. (2017) a privatização do sono, traz consequências graves ao adolescente, tanto de cunho biológico (Ex: complicações no crescimento, modulações hormonais e sensação de exaustão), como também prejuízos sociais (Ex: baixo rendimento escolar, isolamento social, entre outros).

Outro componente comportamental verificado nos estudos foi em relação aos hábitos de higiene bucal dos estudantes. Al Subait AA, et al. (2016) constatou que aproximadamente $67 \%$ dos jovens escolares não escovam devidamente seus dentes. Adicionalmente, Al-Qurashi $\mathrm{H}$, et al. (2016) aponta em seu estudo que aproximadamente $50 \%$ dos adolescentes têm sangramento nas gengivas, sensibilidade dentária e estavam insatisfeitos com a aparência dos seus dentes.

$\mathrm{Na}$ adolescência, é comum a ocorrência de alguns problemas como bulimia que pode levar à erosão dentária e cárie na face lingual dos dentes anteriores, bem como o uso de piercing, que pode causar complicações de ordem inflamatória e até infecciosa, além da má oclusão e mancha nos dentes, halitose (mau hálito), entre outros do campo da estética (BRASIL, 2018).

Ainda no componente comportamental, Meyer MKH, et al. (2017), alerta em sua pesquisa para o uso da espreguiçadeira entre os adolescentes, uma vez encontrada a prevalência de uso nos últimos 12 meses de $22 \%$ dos estudantes, com uma proporção maior entre as mulheres $(29 \%)$ sobre os homens $(12 \%)$.

Os hábitos e comportamentos de saúde devem ocupar um local privilegiado nos debates sobre saúde entre toda a comunidade escolar. Partindo da perspectiva de que o aluno não aprende só ouvindo, mas também observando e executando, a escola precisa ser um local de boas práticas em saúde. Destaca-se aqui ainda a necessidade do desenvolvimento crítico reflexivo do estudante sobre os fatores que o condicionaram a aderir esses comportamentos em suas vidas (MAIA CMMSC, et al., 2017).

Um dos obstáculos mais evidentes à concretização da saúde na escola reside na relutância humana às mudanças de comportamento. Os estudantes compreendem que o espaço escolar auxilia na promoção da saúde, no entanto se percebe a dificuldade de efetivação de um estilo de vida saudável. Recomenda-se, portanto, a qualificação dos profissionais de saúde e o trabalho conjunto com os professores da escola (FAIAL LCM, et al., 2019).

Por fim, trazemos os estudos relacionados à prevalência de vacinação, uso de medicamentos e consumo de drogas pelos estudantes. A pesquisa de Szilagyi PG, et al. (2018), descreve que a taxa geral de vacinação contra o influenza vírus foi maior entre alunos em escolas suburbanas $(51,2 \%$ vs. $45,8 \%, p<0,001)$ e escolas urbanas $(36,7 \%$ vs. $31,4 \%, p=0,01)$. Dessa forma, atribui-se esse resultado a ação da atenção primaria em saúde nos espaços escolares.

Baiden P, et al. (2019) alerta em seu estudo para a prevalência do consumo de álcool na infância, destacando que $15,6 \%$ dos participantes em sua pesquisa começaram a tomar álcool antes dos 13 anos. Outro ponto em destaque é o consumo indevido de esteroides anabolizantes entre os estudantes, no estudo de Jampel JD, et al. (2016) a prevalência do uso foi de de $12,6 \%$ entre os meninos que se consideravam muito abaixo do peso , 11,9\% para os meninos que se consideravam muito acima do peso, comparados com $3,8 \%$ dos meninos que se consideravam com o peso correto. 
Pontuamos ainda, dentro do consumo indevido de compostos pelos estudantes, a prática do tabagismo. Anand V, et al. (2015), traz informações interessantes em seu estudo sobre os cigarros eletrônicos, a prevalência se deu da seguinte forma: A maioria dos estudantes (77,3\%) sabia sobre e-cigarros; $15,2 \%$ relataram que experimentaram um cigarro eletrônico e $60 \%$ relataram que os cigarros eletrônicos eram seguros ou apresentavam riscos mínimos para a saúde. Apenas 5,4\% relataram que as escolas ofereceram informações sobre o uso de cigarros eletrônicos. Zander NC, et al. (2018), complementa ainda que 20,9\% dos adolescentes já experimentaram o tabaco pelo menos uma vez na vida.

O uso dos cigarros eletrônicos avança entre os adolescentes, um produto advento do avanço das novas tecnologias que vem se associando aos hábitos ou comportamentos de risco para a saúde do jovem escolar. Segundo Oliveira MMD, et al. (2017), conhecer como os adolescentes escolares se comportam, sobre os vários aspectos que os circundam, favorece a mensurar a magnitude e a distribuição de importantes fatores de risco à saúde, direcionando informações fundamentais a orientações de políticas públicas. A efetivação de uma vigilância sobre os fatores de risco à saúde na escola minimiza os agravos à saúde nas instituições educacionais e deve ser reforçado com a expectativa de melhorar a qualidade de vida dos discentes.

\section{CONSIDERAÇÕES FINAIS}

Todas as categorias, onde se enquadraram os estudos selecionados nesta revisão, de modo geral, traz indicadores de prevalência de aspectos relacionados com a saúde negativos, que alertam para os perigos e agravos ao bem-estar do estudante adolescente. $O$ estudo nos permite concluir que as atuais medidas de promoção de saúde destinadas ao jovem escolar não estão sendo satisfatórias. E a partir da identificação das carências de saúde diagnosticadas deve-se fundamentar as intervenções futuras. Destaca-se aqui, que carecemos de pesquisas onde o percurso metodológico na coleta de dados se desenvolve de forma logintudinal, o que favoreceria a compreensão dos processos de adoecimento, que sobre uma outra ótica, pode potencializar as intervenções.

\section{REFERÊNCIAS}

1. ALBUHAIRAN F, et al. The relation ship of bullying and physical violence o mental health and academic performance: A crosssectional study among adolescents in Kingdom of Saudi Arabia. International journal of pediatrics and adolescent medicine, 2017: 4(2); 61-65.

2. ALSUBAIE AS, OMER EO. Physical activity be havi or predictors, reasons and barrier samong male adolescents in Riyadh, Saudi Arabia: evidence for obesogenicenvironment. International jornal of health sciences, 2015: 9(4); 400.

3. AL SUBAIT AA, et al. Oral health know ledge, attitude and be havior among students of age 10-18 years old attending Jen adriyah festival Riyadh; a cross-sectional study. The Saudi Journal for Dental Research, 2016: 7(1); 45-50.

4. AL-QURASHI H, et al. Comparison of oral hygiene practices and oral health problems amongs mokerand non-smoker male adolescents in the Eastern Province of Saudi Arabia. The Saudi Journal for Dental Research, 2016: 7(2); 106-111.

5. AMARAL MOP, et al. Quality of life, sleepiness and depressive symptoms in adolescents with insomnia: a cross-sectional study. Atencion primaria, 2017: 49(1); 35-41.

6. ANAND V, et al. E-cigarette use and beliefs among urban public high school students in North Carolina. Journal of Adolescent Health, 2015: 57(1); 46-51

7. ASSIS MM, et al. Consumo alimentar e síndrome metabólica em adolescentes. ScientiaMedica, v. 27, n. 1,2017: 2.

8. BADR HE, et al. Child hood mal treatment: A predictor of mental health problems among adolescents and young adults. Child abuse \& neglect, 2018:80; 161-171.

9. BAIDEN P, et al. Investigating the association between age at first alcohol use and suicidalide ation among high school students: evidence from the you thrisk be havior surveillance system. Journal of affective disorders 2019: 242; 60-67.

10. BALDURSDOTTIR B., et al. Age-related differences in physical activity and depressive symptoms among 10- 19-year-old adolescents: A population based study. Psychology of Sport and Exercise, 2017: 28; 91-99.

11. BARBOSA FNM, et al. C Texto contexto - enferm. , Florianópolis, v. 25, n. 4, e2620015, 2016. Disponível em <http://www.scielo.br/scielo.php?script=sci_arttext\&pid=S0104-07072016000400312\&lng=en\&nrm=iso>. acesso em 26 de março de 2021. Epub, 28 de novembro de 2016. http://dx.doi.org/10.1590/0104-07072016002620015.

12. BECKMAN L, et al. Associations between neuro develop mental disorders and factors related to school, health, and social interaction in schoolchildren: Results from a Swedish population-based survey. Disability and health journal, 2016: 9(4); 663672.

13. BENBENISHTY R, et al. A school-based multilevel study of adolescent suicide ideation in California high schools. The Journal of pediatrics, 2018: 196; 251-257.

14. BRASIL. Ministério da Saúde. Guia alimentar para a população brasileira. 2. ed. Brasília, 2014. 156 p.

15. BRASIL. Secretaria de Atenção à Saúde. Departamento de Ações Programáticas Estratégicas. Proteger e cuidar da saúde de adolescentes na atenção básica. 2. ed. Brasília: Ministério da Saúde, 2018. [acessado 2021 Mar 21]. Disponível em: http://editora.saude.gov.br» http://editora.saude.gov.br.

16. CAMARGO FC, et al. Prática baseada em evidências: revisão bibliométrica das publicações nacionais em periódicos de enfermagem. Revista Família, Ciclos de Vida e Saúde no Contexto Social, 2017: 5(3); 429-439.

17. CHAVES OC, et al. Consumo de refrigerantes e índice de massa corporal em adolescentes brasileiros: Pesquisa Nacional de Saúde do Escolar. Revista Brasileira de Epidemiologia, 2018, 21, e180010. 
18. CLAUDIA CPDSR, et al. Percepção de adolescentes escolares sobre transformações corporais, gravidez e caderneta de saúde do adolescente. Revista Cubana de Enfermería, 2016, 32(1).

19. DE SOUSA DS, PEREIRA RP. Perfil dos fatores de risco para doenças crônicas não-transmissíveis em escolares da zona urbana e rural. Revista Brasileira de Prescrição e Fisiologia do Exercício (RBPFEX), 2018: 12(72); 38-45.

20. DO NASCIMENTO FJ, et al. Sobrepeso e obesidade em adolescentes escolares: uma revisão sistemática. Saúde Coletiva (Barueri), 2020: 10(55); 2947-2958.

21. FAIAL LCM, et al. A escola como campo de promoção à saúde na adolescência: revisão literária. Rev Pró-Univer SUS, Vassouras,2016: 7(2); 22-9.

22. FAIAL LCM, et al. Saúde na escola: percepções de ser adolescente. Rev.Bras. Enferm. Brasília, agosto de 2019: 72(4)964-972.

23. FRANK R, et al. Bodyweight perception and body weight control be haviors in adolescents. Jornal de Pediatria (Versão em Português), 2018: 94(1); 40-47.

24. FONSECA CD, et al. Postural education and be havior among students in a city in southern Brazil: student postural education and be havior. Journal of physical therapy science, 2015: 27(9), 2907-2911.

25. GALVÃO TF, et al. Principais itens para relatar revisões sistemáticas e meta-análises: a recomendação PRISMA. Epidemiologia e Serviços de Saúde, 2015: 24, 335-342.

26. GARCIA-CONTINENTE X, et al. Hábitos alimentarios, conductas sedentarias y sobrepeso y obesidaden adolescentes de Barcelona. Anales de pediatría, 2015: 83(1) 3-1.

27. GUO L, et al. Associations between depression risk, bullying and current smoking among Chinese adolescents: Modulated by gender. Psychiatry research, 2016: 237; 282-289.

28. JARDIM TV, et al. Blood pressure reference alues for Brazilian adolescents: data from the Study of Cardiovascular Risk in Adolescents (ERICA Study). Jornal de pediatria, 2018.

29. JAMIR L, et al. Epidemiology of technology addiction among school students in rural India. Asian journal of psychiatry,2019: 40; 30-38.

30. JAMPEL JD, et al. Self-perceived weight and anabolic steroid misuse among US adolescent boys. Journal of Adolescent Health, 2016: 58(4); 397-402.

31. KAESTNER TL, et al. Prevalence of high cholesterol levels suggestive of familial hyper cholesterolemia in Brazilian adolescents: Data from the study of cardiovascular risk in adolescents. Journal of clinical lipidology, 2018: 1(2) 403-408.

32. KHAN A, et al. Patterns and correlates of physical activity in adolescents in Dhaka city, Bangladesh. Public health, 2017: 145; 75-82.

33. LU L, et al. Internet addiction in Tibetan and Han Chinese middle school students: prevalence, demographics and quality of life. Psychiatry research, 2018: 268; 131-136.

34. MAIA CMMSC, et al. Acompanhamento nutricional por meio da avaliação antropométrica de crianças e adolescentes em uma unidade básica de saúde. Revista Brasileira de Pesquisa em Saúde/Brazilian Journal of Health Research, 2017: 19(1) 28-33.

35. MALTA M. Iniciativa STROBE: subsídios para a comunicação de estudos observacionais. Revista de Saúde Pública, 2010: $44 ; 559-565$.

36. MEYER MKH, et al. Sunbed use among 64,000 Danish students and the associations with demographic factors, health-related be haviours, and appearance-related factors. Preventive medicine, 2017: 100; 17-24.

37. MINATTO G, et al. Health-related physical fitness in Brazilian adolescents from a small town of German colonization. Revista Andaluza de Medicina del Deporte, 2016: 9(2) 67-74.

38. MILLARINI V, et al. Prevalence and co-occurrence of um healthy life style habits and be haviours among secondary school students in Tuscany, central Italy. Public health, 2019: 166; 89-98.

39. MUSA TH, et al. Prevalence of Over weight and Obesity among Students Aged 7-22 Years in Jiangsu Province, China. Biomedical and Environmental Sciences, 2016: 29(10) 697-705.

40. NASCIMENTO BR, et al. Echocardiographic prevalence of rheumatic heart disease in Brazilian school children: Data from the PROVAR study. International jornal of cardiology, 2016: 219; 439-445.

41. OLIVEIRA MMD, et al. Características da Pesquisa Nacional de Saúde do Escolar-PeNSE. Epidemiologia e Serviços de Saúde, 2017: 26; 605-616.

42. O'REILLY M, et al. Revisão das intervenções de promoção da saúde mental nas escolas. Psiquiatria social e epidemiologia psiquiátrica, 2018: 53(7); 647-662.

43. SALES JKD, et al. Fatores de risco associados ao comportamento sexual de adolescentes. Revista Eletrônica Acervo Saúde, 2020: 49, e3382.

44. SANTOS SJD. Association between physical activity, participation in Physical Education classes, and social isolation in adolescents. Jornal de pediatria, 2015: 91(6); 543-550.

45. SAMPASA-KANYINGA $\mathrm{H}$, et al. Sleep duration and consumption of sugar-sweetened bever ages and energy drinks among adolescents. Nutrition, 2018: 48; 77-81.

46. SHARMA B, et al. Prevalence and correlates of insufficient physical activity in school adolescents in Peru. Revista de saude publica, 2018: 52; 51.

47. SILVA FMDA, et al. Consumption of fruits and vegetables associated with other isk be haviors among adolescents in Northeast Brazil. Revista Paulista de Pediatria, 2016: 34(3) 309-315.

48. SOUZA AA, CADETE MMM, O papel das famílias e da escola na formação de hábitos alimentares saudáveis de crianças escolares. Revista Pedagógica, 2017 jan./abr, 19(40).

49. SZILAGYI PG, et al. School-located influenza vaccinations for adolescents: a randomized controlled trial. Journal of Adolescent Health, 2018: 62(2); 157-163.

50. VANCAMPFORT D, et al. Sedentary be havior and depressive symptoms among 67,077 adolescentsaged12-15 years from 30 low-and middle-income countries. International Journal of Be havioral Nutrition and Physical Activity, 2018: $15(1) ; 73$.

51. VANCAMPFORT D, et al. Leisure-time sedentary be havior and suicide attempt among 126,392 adolescents in 43 countries. Journal of affective disorders, 2019.

52. VILELA TDS, et al. Factors influencing excessive daytimes leepiness in adolescents. Jornal de pediatria, 2016: 92(2); 149155.

53. YANG L, et al. Physical fighting and associated factors among adolescents aged 13-15 years in six western Pacific countries. International jornal of environ mental research and publich ealth, 2017:14(11); 1427.

54. YANG L, et al., Prevalence of under weight and over weight among young adolescents aged 12-15 years in 58 low-income and middle-income countries. Pediatric obesity, 2019: 14(3) e12468.

55. ZANDER NC, et al. Tabaco en adolescentes escolares brasileños: asociación con salud mental y contexto familiar. Gaceta Sanitaria, 2018: 32; 216-222. 